\title{
Zur Frage des Einflusses psychischer Depressionen und der Vorstellung schwerer Muskelarbeit auf den Stofiwechsel.
}

\author{
(Untersuchungen in der Hypnose.) \\ Von
}

Prof. E. Grafe und Dr. E. Traumann

in Gemeinschaft mit den Herren cand. med. Laufer und Samson

(Aus der Medizinischen Klinik zu Heidelberg.)

(Eingegangen am 22. August 1920.)

Es ist eine bekannte Erfahrung des täglichen Lebens, daß Sorge zehrt, d.h. zu erheblichen Gewichtsabnahmen führt. Besonders eindrucksvolle Beispiele dafür haben wir in den Kriegsjahren erlebt. Natürlich kommen hier nur solehe Menschen in Betracht, bei denen die Ernährungsverhältnisse und auch die Nahrungsaufnahme gleich oder ähnlich günstig waren wie vor dem Kriege. Die Beurteilung derartiger Dinge ist darum sehr erschwert, weil im einzelnen Falle natürlich nicht genau bekannt ist, ob die Angabe, daß tatsächlich genau so viel Nahrung aufgenommen wurde wie früher, richtig war; meist ist die Hauptursache der Gewichtsabnahme bei Kummer und Sorge Daniederliegen des Appetits und demzufolge mangelhafte Nahrungsaufnahme. Grafe ${ }^{1}$ ) beobachtete bei einem Arzte, der vollkommen im Stoffwechselgleichgewicht war (tägliche Feststellung des Körpergewichtes und der ihrer Zusammensetzung nach genau bekannten Nahrung), daß unter dem Einfluß eines schweren Schicksalsschlages, der ihn und seine Familie getroffen hatte, im Laufe weniger Tage bei gleich großer Nahrungsaufnahme eine Gewichtsabnahme von $2 \mathrm{~kg}$ eintrat. Solche Beobachtungen legen nun den Gedanken nahe, ob nicht schwere Gemütsbewegungen zu einer Erhöhung des Stoffwechsels führen können. Irgendein sicherer Beweis, daß derartige Dinge vorkommen, fehlt zur Zeit völlig. Am meisten würden sich zur Entscheidung dieser Frage psychisch Schwerkranke, Melancholiker, Depressions zustände usw. eignen. Hier könnte entweder in der Weise vorgegangen werden, daß in einer langen Versuchsperiode festgestellt wird, mit welchem Caloriengehalt der Nahrung noch Gewichtskonstanz erzielt werden kann. So lassen sich wenigstens ungefähr Anhaltspunkte

1) Nicht veröffentlichte Versuche mit Dr. W. Röhmer. 
gewinnen. Oder aber, und das ist der allein sichere Weg, es werden bei diesen Kranken lang dauernde Respirationsversuche vorgenommen. Die erste Methode ist von den Psychiatern ${ }^{1}$ ) immer noch nicht in größerem Maßstabe angewandt worden, weil man sich scheut, solche Kranke ohne genügenden Grund gewaltsam zu ernähren. Gegen die Untersuchung im Respirationsapparat wird stets das Bedenken geltend gemacht, daß der Aufenthalt in der engen, ungewohnten Kammer auf das psychische Befinden der Kranken leicht ungünstig wirken und so eine Verschlimmerung des Krankheitszustandes herbeiführen könnte.

Grafe $e^{2}$ ) hat seinerzeit über das Verhalten des respiratorischen Gaswechsels bei Stuporen in lang dauernden Versuchen Untersuchungen mitgeteilt, aus denen hervorgeht, daß in sehr vielen Fällen erhebliche Herabsetzung des Stoffwechsels vorhanden war, und zwar betrafen die Herabsetzungen vorwiegend die Fälle von katatonischem Stupor, während bei manisch depressivem Stupor dies nicht sicher war, hier sogar geringe Stoffwechselsteigerungen vorkommen konnten. Aber auch diese Untersuchungen sind zur Beantwortung der eben aufgeworfenen Frage nicht geeignet, da einmal die psychiatrische Diagnose in vielen Fällen unsicher ist und zweitens der seelische Inhalt der Kranken während des Stupors nicht sicher bekannt ist.

Da in der geschilderten Weise eine Entscheidung nicht zu treffen war, so konnte man daran denken, auf dem Wege der Hypnose schwere Depressionszustände zu erzeugen und direkt beim Gesunden, wo exakte Vergleichsperioden beim gleichen Individuum zur Verfügung standen, den Einfluß suggestiv erzeugter schwerer seelischer Aufregungen zu untersuchen. Dieser Frage, die in Gemeinschaft mit den Herren cand. med. Laufer und Samson in Selbstversuchen bei diesen Herren bearbeitet wurde, gelten die folgenden Versuche.

Úber den Einfluß der Affekte auf den respiratorischen Gaswechsel fehlen unseres Wissens in der Literatur Versuche bisher vollständig. Dagegen ist der Einfluß geistiger Arbeit auf den Stoffwechsel schon von Lavoisier zum Gegenstand von Untersuchungen gemacht worden. Seitdem ist diese Frage oft diskutiert, seltener bearbeitet worden. Erwähnt seien aus älterer Zeit ${ }^{3}$ ) die Mitteilungen von Kraft Ebing, Liebermeister, Löwy, Speck, Atwater, Woods und Benedict, Richet, Johannsson, Zu ntz und Löw y. Nur die beiden ersten Untersucher sind geneigt, eine Stoffwechselsteigerung durch geistige Arbeit anzunehmen. Aus jüngster Zeit liegen die wichtigen umfassenden Unter-

1) Rosenfeld hat bei Katatonikern ähnliche Versuche vorgenommen, dort auch die ältere Literatur. Zeitschr. f. Psychiatrie 63, 36. 1906.

2) Deutsch. Arch. f. klin. Med. 102, 15. 1911. Dort auch die ältere Literatur.

s) Genaue Literatur bei F. C. Becker und O. Olsen. Skand. Arch. f. Physiol.

31, 81. 1914 und bei Rosenfeld 1. c. 
suchungen von Benedict und Carpenter ${ }^{1}$ ) sowie Becker und Olse $n^{2}$ ) vor. Benedict und Carpenter vermochten keine Steigerung nachzuweisen, während die dänischen Forscher in Lehmanns Laboratorium zu dem Resultate kamen, daß doch eine Erhöhung vorhanden ist. Diese kam in dem ersten Versuche am stärksten zum Ausdruck und ließ dann in dem Maße, wie die Versuchspersonen an die ihnen aufgetragene geistige Arbeit (Addieren und Multiplizieren) sich gewöhnt hatten, nach. Die Versuchsresultate sind in hohem Grade abhängig von der angewandten Methodik, auf deren Kritik hier nicht eingegangen werden soll. Jedenfalls ist auch die Frage des Einflusses geistiger Arbeit auf den respiratorischen Gaswechsel noch nicht definitiv geklärt.

Für die Beantwortung der Frage, ob hypnotisch erzeugte Depressionen einen Einfluß auf den respiratorischen Stoffwechsel haben, kommen nur etwas länger dauernde Versuche in Betracht, da mehrere Stunden nötig sind, um eine eventuelle Einwirkung auf die Wärmeproduktion zur Geltung zu bringen.

Die Versuchspersonen, die Herrn cand. med. La ufer und Samson, welche sich mit großem Interesse und außerordentlicher Gewissenhaftigkeit diesen Untersuchungen unterzogen, waren körperlich und geistig vollkommen gesund. Sie kamen jeweils für genau 2 Stunden in den Respirationsapparat der Klinik, der nach Ja que te Prinzip von dem einen von uns $\left.(G .)^{3}\right)$ konst ruiert war. Bei allen Versuchen wurde streng darauf geachtet, daß 12 - 13 Stunden vorher keine Nahrung mehr aufgenommen war und daß schon $1 / 4-1 / 2$ Stunde vorher und während der Versuche strenge Muskelruhe eingehalten, auch Spannungen vermieden wurden. In dieser Richtung wurde auch während der Versuche noch häufiger besondere Suggestionen gegeben. Die Hypnosen wurden von dem einen von uns (Dr. Traumann), der im Neurosenlazarett Hornberg während $1 \frac{1}{4}$ jähriger Tätigkeit eine genügende Erfahrung auf hypnotischen Gebiete gewonnen hatte, vorgenommen. Die eigentlichen Untersuchungen wurden erst begonnen, nachdem in zahlreichen Sitzungen dio Eignung der Versuchspersonen mit Sicherheit festgestellt und die suggestive Kraft des Hypnotiseurs gesichert war. Während der Hypnose wurde in kleineren Zeitabständen außerhalb des Apparates Puls, Temperatur, Atmung und in mehreren Versuchen auch der Blutdruck gemessen. Die Kontrolle von Puls- und Blutdruck wurde in der Weise vorgenommen, daß die Recklinghausensche Manschette mit dem Recklinghausenschen Blutdruckapparat durch einen langen Schlauch, der durch eine Wandöffnung der Kammer luftdicht durchgezogen war, verbunden wurde. An den Ausschlägen des Manometers konnte neben dem Blutdruck, dessen Bestimmung allerdings nicht immer genügend scharf gelang, gleichzeitig auch die Pulsfrequenz festgestellt werden. Selbstverständlich wurden vor und nach der Hypnose ebenfalls die einschlägigen Faktoren quantitativ festgestellt.

1) U. S. Dept. of Agric. Offic. of Experim. Stations-Bull. Nr. 208, vgl. auch Benedict, The influence of mental and Muscular Work on nutritive Process. imprint. from Proceed. of the Americ. Philosoph. Society 49, 195. 1910.

2) 1 . c.

3) Über die Technik vgl. Grafe, Die Technik der Untersuchung des respiratorischen Gaswechsels beim gesunden und kranken Menschen in Abderhaldens Handbuch der biochemischen Arbeitsmethoden Bd. VII. 1913. 
Um auch Anhaltspunkte für die Eiweißverbrennung zu haben, insbesondere auch die Calorienproduktion exakt berechnen zu können, wurde während der Versuchsperiode der Urin gesammelt und sein Stickstoffgehalt nach Kjeldahl bestimmt. Die Berechnung der Calorienproduktion auf Grund der respiratorisch gefundenen Werte für $\mathrm{CO}_{2}$ und $\mathrm{O}_{2}$, sowie $\mathrm{N}$ im Harn geschah in der von $\mathrm{Zuntz}{ }^{1}$ ) angegebenen Weise.

Da der Einfluß der Hypnose auf den respiratorischen Stoffwechsel anscheinend überhaupt noch nicht untersucht worden ist, so war zunächst von Interesse festzustellen, wie der hypnotisch erzeugte Schlaf einmal gegenüber dem Normalschlaf und zweitens gegenüber dem Zustand vorsätzlicher Muskelruhe in wachem Zustand sich verhielt. Die Versuche mit hypnotischem Schlaf dienten zum Vergleich und als Basis für die Versuche mit Erzeugung einer Depression. Sie wurden zeitlich möglichst nahe an die anderen herangelegt, an einzelnen Tagen auch direkt mit den anderen verbunden, was allerdings zu starker Ermüdung führte und sich im allgemeinen nicht empfiehlt. Selbstverständlich befand sich der Hypnotiseur während der Hypnose dauernd außerhalb des Kastens neben dem mit großen Fenstern versehenen Respirationsapparate, so daß eine permanente Einwirkung vorhanden war. Utber die Art der Versuchsanlage orientieren im einzelnen die als Beispiel im Anhang mitgeteilten Protokolle ${ }^{2}$ ), die hauptsächlich die fortlaufenden Angaben, Beobachtungen und Messungen des Hypnotiseurs umfassen, denen aber außerdem die Eindrücke, die der Hypnotisierte selbst von der Hypnose bzw. von den Wirkungen der Hypnose gehabt hat, beigefügt sind.

Die Ergebnisse der mitgeteilten Versuche bezüglich des Stoffwechsels sind in der großen Ubersichtstabelle zusammengefaßt. Stab 6 enthält die Angabe über die Art des Versuches und vor allen Dingen den Inhalt der Suggestion. Stab 7-10 die wichtigsten Angaben über Körpertemperatur, Pulsfrequenz, Blutdruck und Respiration. Utber das Verhalten der Motilität ist in Stab 11 nur eine kurze Zusammenfassung gegeben, die das Wichtigste aus den genauen Protokollen der Einzelversuche ergibt. Gerade auf diesen Punkt wurde ganz besonders geachtet, und da sowieso ja in den Untersuchungen nur kleine Ausschläge zu erwarten waren, mußte gerade die Kontrolle der Muskelbewegung möglichst exakt durchgeführt werden. Stab 12-14 bringen die Werte für die $\mathrm{CO}_{2}$ - und $\mathrm{O}_{2}$-Menge in Litern, wie sie im zweistündigen Versuch festgestellt waren, sowie die Reduktion auf Körpergewicht und Minute. Stab 15 das Verhalten des Urins nach Menge und Stickstoffgehalt, 16 die Calorienproduktion, sowie Stab 17 das Verhalten gegenüber der Vergleichsperiode ausgedrückt in Prozenten. Um schließlich die Ver-

1) Vgl. z. B. sein Lehrbuch der Physiol. gemeinsam mit Löw y, erste Aufl., S. 661.1909.

2) Die gesamten Protokolle finden sich ausführlich in der Inauguraldissertation von R. Laufer, Stoffwechseluntersuchungen in der Hypnose. Heidelberg 1920. 
suche alle untereinander zu vergleichen, wurden in Stab 18 die Calorien pro Körperkilogramm und 2 Stunden umgerechnet.

Was zunächst die Frage des Einflusses des hypnotischen Schlafes auf den Stoffwechsel gegenüber dem normalen Grundumsatzversuch angeht, so stehen hierfür die Versuche 1, 2, 5, 6, 10, 13 und 18 zur Verfügung. Aus ihnen geht hervor, daß der hypnotische Schlaf gegenüber dem Grundumsatz stets zu einem Absinken des Stoffwechsels führt, der $5,2-9,9 \%$ beträgt (Versuche 6 und 13). Nur im Versuch 1 und 2 trat dies nicht hervor, aber dabei ist $\mathrm{zu}$ bedenken, daß dies die beiden ersten Versuche waren, und daß außerdem hier nur die Kohlensäure bestimmt worden war.

Von Interesse ist, daß der gewöhnliche Schlaf (Vers. Nr. 8) gegenüber dem hypnotischen (Vers. Nr. 6) zu einer geringen Steigerung von 3,2\% führt. Allerdings ist der Unterschied so gering, daß die Differenz vielleicht überhaupt in den Bereich der biologischen Fehlerquellen fällt. Möglicherweise ist sie damit zu erklären, daß beim normalen Schlaf völlige Körperruhe nicht in dem Maße zu erzielen ist wie in der Hypnose, wo stets noch besondere Suggestionen, bewegungslos stillzuliegen, gegeben wurden. Auf Grund dieses einen Versuchs läßt sich die skizzierte Frage natürlich nicht entscheiden.

Der Inhalt der in den Depressionsversuchen gegebenen Suggestionen ist aus den Angaben in Stab 6 ersichtlich. Selbstverständlich wußte die Versuchsperson niemals vorher etwas von dem Versuchsplane, so daß eine seelische Einstellung vorher nicht in Betracht kommt.

Für die Frage, ob die hypnotische Erzeugung einer schweren Depression einen Einfluß auf den respiratorischen Stoffwechsel hat, kommen bei $\mathrm{L}$. die Versuche 4, 9, 12 sowie 19 in Betracht. Wie z. B. aus dem hinten angeschlossenen Protokoll (S. 250, Nr. 4) hervorgeht, war die Wirkung der Suggestion auf die Stimmungslage keine ganz ungehemmte, da sowohl in dem Versuch Nr. 4, wo die tödliche Erkrankung an einem inoperablen Magencarcinom suggeriert wurde, wie bei dem Versuch Nr. 9, Gegensuggestionen sich geltend machten; nur in dem letzten Versuch, in dem die Erkrankung an Hirntumor suggeriert wurde, fielen Störungen dieser Art fort. Nur in einem dieser Versuche: Nr. 9, kam es zu einer ganz geringen Steigerung des Stoffwechsels gegenüber dem 3 Tage vorherliegenden Vergleichsversuch mit hypnotischem Schlaf allein. Die Stoffwechselsteigerung ist aber erst recht außerordentlich gering, wenn man statt des Versuches Nr. 7 als Vergleichsversuch den Versuch $\mathrm{Nr}: 10$, der allerdings 9 Tage später stattfand, in Betracht zieht. In diesem Falle würde die Steigerung sogar unter $1 \%$ fallen. Analog liegen die Verhältnisse beim 4. Versuche. In den anderen Versuchen 12 und 19 war ein geringes Absinken des Stoffwechsels vorhanden; im ersteren Falle von $5,3 \%$ und im zweiten Falle 
242 E. Grafe u. E. Traumann: Zur Frage des Einflusses psychischer Depressionen

\begin{tabular}{|c|c|c|c|c|c|c|c|c|c|}
\hline 1 & 2 & $\mathbf{3}$ & 4 & 5 & 6 & 7 & 8 & 9 & 10 \\
\hline 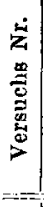 & 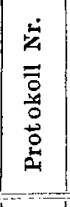 & $\begin{array}{c}\text { Datum } \\
1919\end{array}$ & $\begin{array}{c}\text { Ver- } \\
\text { suchs- } \\
\text { person } \\
\text { (Körper- } \\
\text { größe) }\end{array}$ & \begin{tabular}{|c|} 
Körper- \\
gewicht \\
$\mathrm{kg}$
\end{tabular} & Art des Versuchs & $\begin{array}{c}\text { Mittelwert } \\
\text { derKörper- } \\
\text { tempera- } \\
\text { tur(Außen- } \\
\text { tempera- } \\
\text { tur im } \\
\text { Kasten) }\end{array}$ & $\begin{array}{c}\text { Mittelwert } \\
\text { der Puls- } \\
\text { frequenz } \\
\\
\text { (mi-ma) }\end{array}$ & $\begin{array}{l}\text { Mittelwert } \\
\text { des Blut- } \\
\text { druckes } \\
\text { (mi-ma) }\end{array}$ & $\begin{array}{l}\text { Mittelwert } \\
\text { der Respi- } \\
\text { rationsfre- } \\
\text { quenz } \\
\text { (mi - ma) }\end{array}$ \\
\hline 1 & 346 & 3. IX. & $\begin{array}{l}\text { Laufer } \\
(165 \mathrm{~cm})\end{array}$ & 66,350 & $\begin{array}{c}\text { Grundumsatzversuch bei vorsätz- } \\
\text { licher Muskelruhe und Nüchtern- } \\
\text { heit }\end{array}$ & $\begin{array}{r}36,2^{\circ} \\
\left(18,5^{\circ}\right)\end{array}$ & 60 & - & 14 \\
\hline 2 & 347 & 4. IX. & $"$ & 66,200 & Hypnotischer Schlaf & $\begin{array}{l}36,3^{\circ} \\
\left(20,7^{\circ}\right)\end{array}$ & $\begin{array}{c}58 \\
(54-60)\end{array}$ & $\begin{array}{l}110 \mathrm{~mm} \mathrm{Hg} \\
(110-110)\end{array}$ & $\left(\begin{array}{c}16 \\
(12-20)\end{array}\right.$ \\
\hline 3 & 348 & 5. IX. & $"$ & 66,100 & $\begin{array}{c}\text { Hypnose mit Suggestion einer } \\
\text { schweren körperlichen Anstren- } \\
\text { gung (Rudern, Radfahren, Ge- } \\
\text { wichtheben) }\end{array}$ & $\begin{array}{c}36,1^{c} \\
\left\{20,6^{\circ}\right\rangle\end{array}$ & $\begin{array}{c}56 \\
(44-68)\end{array}$ & $\begin{array}{c}166 \mathrm{H}_{2} \mathrm{O} \\
(150-180)\end{array}$ & $\left(10^{27}-48\right)$ \\
\hline 4 & 349 & 6. IX. & $"$ & 66,000 & $\begin{array}{c}\text { Hypnose mit der Suggestion einer } \\
\text { schweren psychischen Depressiun } \\
\text { (Erkrankung an inoperablem Ma- } \\
\text { gencarcinom) }\end{array}$ & $\begin{array}{c}36,3^{\circ} \\
\left(21,2^{\circ}\right)\end{array}$ & $(48-66)$ & $\begin{array}{c}170 \mathrm{H}_{2} \mathrm{O} \\
(170-190)\end{array}$ & $\begin{array}{c}19 \\
(15-24)\end{array}$ \\
\hline 5 & 350 & 9. IX. & 11 & 66,000 & $\begin{array}{c}\text { Grundumsatzversuch bei vorsätz- } \\
\text { licher Muskelruhe und Nüchtern- } \\
\text { heit }\end{array}$ & $\begin{array}{r}36,0^{\circ} \\
\left(20,9^{\circ}\right) \\
\end{array}$ & $\mid \begin{array}{c}53 \\
(50-57)\end{array}$ & 一 & $\begin{array}{c}17 \\
(16-19)\end{array}$ \\
\hline 6 & 351 & 10. IX. & 9 & 66,200 & Hypnotischer Schlaf & $\begin{array}{l}36,0^{\circ} \\
\left(21,2^{\circ}\right)\end{array}$ & $\left|\begin{array}{c}50 \\
(46-59)\end{array}\right|$ & $\mid \begin{array}{c}159 \mathrm{H}_{2} \mathrm{O} \\
(147--182)\end{array}$ & $\left(\begin{array}{c}16 \\
(15-18)\end{array}\right.$ \\
\hline 7 & 352 & 11. IX. & $"$ & 66,300 & $\begin{array}{l}\text { Hypnose mit Suggestion einer } \\
\text { schweren körperlichen Arbeit } \\
\text { (Rudern, Gewichtstemmen) }\end{array}$ & $\begin{array}{c}36,15^{\circ} \\
\left(21,55^{\circ}\right)\end{array}$ & $\begin{array}{c}49 \\
(46-52)\end{array}$ & $\mid \begin{array}{c}174 \mathrm{H}_{2} \mathrm{O} \\
(168-185)\end{array}$ & $\begin{array}{c}22 \\
(10-30)\end{array}$ \\
\hline 8 & 353 & 12.IX. & $n$ & 65,800 & Gewöhnlicher Schlaf & $\begin{array}{c}36,1^{\circ} \\
\left(20,9^{\circ}\right)\end{array}$ & $\begin{array}{c}52 \\
(50-56)\end{array}$ & $178 \mathrm{H}_{2} \mathrm{O}$ & $\begin{array}{c}17 \\
(15-19)\end{array}$ \\
\hline 9 & 354 & 13.IX. & $n$ & 65,000 & $\begin{array}{c}\text { Hypnose mit Suggestion einer } \\
\text { schweren psychischen Depression } \\
\text { (Vermögensverlust, Gefangen- } \\
\text { nahme) }\end{array}$ & $\begin{array}{c}36,38^{\circ} \\
\left(21,4^{\circ}\right)\end{array}$ & $\begin{array}{c}49 \\
(47-58)\end{array}$ & $\begin{array}{c}178 \mathrm{H}_{2} \mathrm{O} \\
(17 \overline{5}-180)\end{array}$ & $\begin{array}{c}19 \\
(12-25)\end{array}$ \\
\hline 10 & 359 & 22. IX. & $"$ & 65,050 & Schlafhypnose & $\begin{array}{r}35,85^{\circ} \\
\left(15,0^{\circ}\right)\end{array}$ & $\begin{array}{c}54 \\
(50-66)\end{array}$ & $\begin{array}{c}170 \mathrm{H}_{2} \mathrm{O} \\
-\end{array}$ & $\begin{array}{c}16 \\
(13--20)\end{array}$ \\
\hline 11 & 360 & 23. IX. & $" 1$ & 65,450 & $\begin{array}{l}\text { Hypnose mit Suggestion einer } \\
\text { starken körperlichen Anstrengung } \\
\text { (Gewichtstemmen, Rudern) }\end{array}$ & $\begin{array}{c}36,15 \\
\left(15,8^{\circ}\right)\end{array}$ & $\begin{array}{c}52 \\
(48-68)\end{array}$ & - & $\left(\begin{array}{c}24 \\
(13-29)\end{array}\right.$ \\
\hline
\end{tabular}


und der Vorstellung schwerer Muskelarbeit auf den Stoffwechsel.

\begin{tabular}{|c|c|c|c|c|c|c|c|c|}
\hline 11 & 12 & 18 & 11 & 15 & 16 & 17 & 18 & 19 \\
\hline Verhalten der Motilität & $\begin{array}{l}1 \mathrm{CO}_{2} \text {-Bildung } \\
\text { im Versuch. } \\
{\left[120^{\prime}\right]} \\
\text { (ccm pro } \mathrm{kg} \\
\text { und Minute) }\end{array}$ & $\begin{array}{l}1 \mathrm{O}_{2} \text {-Ver- } \\
\text { brauch imVer- } \\
\text { such. }\left[120^{\prime}\right] \\
\text { (ccm pro } \mathrm{kg} \\
\text { und Minute) }\end{array}$ & R.-Q. & $\begin{array}{l}\text { g N im Ver- } \\
\text { such (ccm } \\
\text { Urin) auf } 2 \\
\text { Stunden } \\
\text { umge- } \\
\text { rechnet }\end{array}$ & \begin{tabular}{|} 
Calo- \\
rienpro- \\
duktion \\
im Ver- \\
such
\end{tabular} & $\begin{array}{c}\text { Veränderung in } \\
\text { Proz.gegenüber } \\
\text { dem Vergleichs- } \\
\text { versuch }\end{array}$ & \begin{tabular}{|l|} 
Calo- \\
rien \\
pro kg \\
und 2 \\
Stun- \\
den
\end{tabular} & $\begin{array}{l}\text { Bemer- } \\
\text { kungen }\end{array}$ \\
\hline $\begin{array}{l}\text { Dauernd fast vollkommen } \\
\text { bewegungslos und ent- } \\
\text { spannt, nicht schlafend }\end{array}$ & $\begin{array}{c}23,571 \\
(2,96 \mathrm{ccm})\end{array}$ & - & 一 & $\begin{array}{c}0,351 \mathrm{~g} \\
(565 \mathrm{ccm})\end{array}$ & - & - & 一 & 一 \\
\hline $\begin{array}{l}\text { Während der } 2 \text { Stunden } \\
\text { keinerlei Bewegungen }\end{array}$ & $\begin{array}{c}24,571 \\
(3,09 \mathrm{ccm})\end{array}$ & - & - & $\begin{array}{c}0,725 \mathrm{~g} \\
(320 \mathrm{ccm})\end{array}$ & - & - & - & 一 \\
\hline $\begin{array}{l}\text { Lecken der Lippen, einmal } \\
\text { Bewegung des rechten } \\
\text { Armes.Öfters Kopfschütteln }\end{array}$ & $\begin{array}{c}27,621 \\
(3,48 \mathrm{~cm})\end{array}$ & $\begin{array}{c}27,81 \\
(3,50 \mathrm{~cm})\end{array}$ & 0,994 & $\begin{array}{c}1,104 \mathrm{~g} \\
(655 \mathrm{ccm})\end{array}$ & 134,4 & + c. $12,4 \%$ & 2,03 & $\begin{array}{l}\text { Am Abend } \\
\text { rorher } \\
\text { reichliche } \\
\text { Kohlenhy- } \\
\text { dratnah- } \\
\text { rung }\end{array}$ \\
\hline $\begin{array}{l}\text { Auf Befragen einmal Kopf- } \\
\text { nicken }\end{array}$ & $\begin{array}{c}23,61 \mathrm{l} \\
(2,99 \mathrm{ccm})\end{array}$ & $\begin{array}{c}24,41 \\
(3,08 \mathrm{ccm})\end{array}$ & 0,970 & $\left\{\begin{array}{c}0,918 \mathrm{~g} \\
(285 \mathrm{ccm})\end{array}\right.$ & 118,85 & $\begin{array}{c}(-3,9 \% \\
\text { gegenüb.Vers. } 2) \\
+4,6 \% \\
\text { gegenunb.Vers. } 6\end{array}$ & 1,80 & $n$ \\
\hline $\begin{array}{l}\text { Dauernd ganz ruhig, nicht } \\
\text { schlafend }\end{array}$ & $\begin{array}{c}23,62 \mathrm{l} \\
(2,98 \mathrm{ccm})\end{array}$ & $\begin{array}{c}24,41 \\
(3,08 \mathrm{ccm})\end{array}$ & 0,968 & $\begin{array}{c}0,863 \mathrm{~g} \\
(120 \mathrm{ccm})\end{array}$ & 119,8 & - & 1,82 & $n$ \\
\hline $\begin{array}{l}\text { Eine kleine Bewegung des } \\
\text { Zeigefingers und einmal } \\
\text { Scblucken; mit dem Kopfe } \\
\text { einzelne leichte Bewe- } \\
\text { gungen }\end{array}$ & $\begin{array}{c}23,091 \\
(2,91 \mathrm{ccm})\end{array}$ & $\begin{array}{c}23,31 \\
(2,93 \mathrm{~cm})\end{array}$ & 0,991 & $\begin{array}{c}1,095 \mathrm{~g} \\
(260 \mathrm{ccm})\end{array}$ & 113,6 & $\mid \begin{array}{c}-5,2 \% \text { ge- } \\
\text { genuber dem } \\
5 . \text { Versuch }\end{array}$ & 1,72 & $"$ \\
\hline $\begin{array}{l}\text { Auf Befehl Kopfnicken. } \\
\text { Leichte Daumenbewegung } \\
\text { und Kopfbewegung rück- } \\
\text { wärts, Lecken der Zunge }\end{array}$ & $\begin{array}{c}23,341 \\
(2,93 \mathrm{ccm})\end{array}$ & $\begin{array}{c}24,31 \\
(3,05 \mathrm{ccm})\end{array}$ & 0,961 & $\begin{array}{c}1,314 \mathrm{~g} \\
(225 \mathrm{~cm})\end{array}$ & 118 & $\begin{array}{c}+3,8 \% \text { ge- } \\
\text { genüber dem } \\
6 . \text { Versuch }\end{array}$ & 1,78 & $"$ \\
\hline- & $\begin{array}{c}22,271 \\
(2,81 \mathrm{ccm})\end{array}$ & $\begin{array}{c}24,2 \mathrm{l} \\
(3,065 \mathrm{~cm})\end{array}$ & 0,920 & $\begin{array}{c}0,840 \mathrm{~g} \\
(245 \mathrm{ccm})\end{array}$ & 117,3 & $\begin{array}{c}+3,2 \% \text { ge- } \\
\text { genuber dem } \\
\text { hypnotischen } \\
\text { Schlaf }\end{array}$ & 1,78 & $"$ \\
\hline $\begin{array}{l}\text { Auf Fragen Kopfnicken und } \\
\text { Kopfschitteln. Auf Befehl } \\
\text { Stellungsänderung einer } \\
\text { Hand. Verziehen des Ge- } \\
\text { sichts }\end{array}$ & $\left(\begin{array}{c}20,03 \mathrm{I} \\
(2,57 \mathrm{~cm})\end{array}\right.$ & $\begin{array}{c}24,91 \\
(3,19 \mathrm{ccm})\end{array}$ & 0,803 & $\begin{array}{l}0,748 \mathrm{~g} \\
(54 \mathrm{ccm})\end{array}$ & 119,6 & $\begin{array}{c}+5,3 \% \text { ge- } \\
\text { gen.Versuch } 6 \\
+0.58 \% \text { ge- } \\
\text { gen. Versuch } \\
10\end{array}$ & 1,84 & 一 \\
\hline $\begin{array}{l}\text { Abwehrbewegungen und } \\
\text { Verdrehen des Gesichtes } \\
\text { wegen einer im Kasten be- } \\
\text { findlichen Fliege }\end{array}$ & $\begin{array}{c}22,145 \mathrm{l} \\
(2,84 \mathrm{ccm})\end{array}$ & $\begin{array}{c}25,21 \\
(3,23 \mathrm{ccm})\end{array}$ & 0,879 & $\begin{array}{c}1,015 \mathrm{~g} \\
(205 \mathrm{ccm})\end{array}$ & 118,9 & $\begin{array}{l}-9,9 \% \text { ge- } \\
\text { genüber dem } \\
13 . \text { Versuch }\end{array}$ & 1,83 & 一 \\
\hline $\begin{array}{l}\text { Auf Befehl Kopfnicken; } \\
\text { sonst keinerlei Körper- } \\
\text { bewegungen }\end{array}$ & $\begin{array}{c}22,631 \\
(2,88 \mathrm{ccm})\end{array}$ & $\begin{array}{c}25,71 \\
(3,27 \mathrm{~cm})\end{array}$ & 0,880 & $\begin{array}{c}0,846 \mathrm{~g} \\
(230 \mathrm{ccm})\end{array}$ & 120,9 & $+1,7 \%$ & 1,87 & - \\
\hline
\end{tabular}


244 E. Grafe u. E. Traumann : Zur Frage des Einflusses psychischer Depressionen

\begin{tabular}{|c|c|c|c|c|c|c|c|c|c|}
\hline 1 & 2 & 3 & 4 & 5 & 6 & 7 & 8 & 9 & 10 \\
\hline 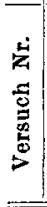 & 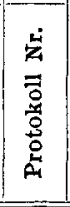 & $\begin{array}{c}\text { Datum } \\
1919\end{array}$ & $\begin{array}{l}\text { Ver- } \\
\text { suchs- } \\
\text { person } \\
\text { (Körper- } \\
\text { größe) }\end{array}$ & $\left|\begin{array}{c}\text { Körper- } \\
\text { gewicht } \\
\mathrm{kg}\end{array}\right|$ & Art des Versuchs & \begin{tabular}{|c|} 
Mittelwert \\
derKörper- \\
tempera- \\
tur(Außen- \\
tempera- \\
tur im \\
Kasten) \\
\end{tabular} & $\begin{array}{l}\text { Mittelwert } \\
\text { der Puls- } \\
\text { frequenz } \\
\text { (mi - ma) }\end{array}$ & $\begin{array}{c}\text { Mittelwert } \\
\text { des Blut- } \\
\text { druckes } \\
\text { (mi-ma) }\end{array}$ & $\begin{array}{l}\text { Mittelwert } \\
\text { der Respi- } \\
\text { rationsfre- } \\
\text { quenz } \\
\\
(\mathrm{mi}-\mathrm{ma})\end{array}$ \\
\hline 12 & 361 & 24. IX. & $\begin{array}{c}\text { Laufer } \\
(165 \mathrm{~cm})\end{array}$ & 64,700 & $\begin{array}{c}\text { Hypnose mit Suggestion einer } \\
\text { schweren seelischen Depression } \\
\text { (Verschüttung) }\end{array}$ & $\begin{array}{c}36,1^{\circ} \\
\left(17,3^{\circ}\right)\end{array}$ & $\begin{array}{c}50 \\
(46-52)\end{array}$ & $\left|\begin{array}{c}107,5 \mathrm{Hg} \\
(105-110)\end{array}\right|$ & $\begin{array}{c}16 \\
(13-19)\end{array}$ \\
\hline 13 & 362 & 25. IX. & $"$ & 64,700 & $\begin{array}{l}\text { Grundumsatzversuch bei vorsätz- } \\
\text { licher Muskelruhe und Nüchtern- } \\
\text { heit }\end{array}$ & $\begin{array}{r}36,0^{\circ} \\
\left(18,2^{\circ}\right)\end{array}$ & $\begin{array}{c}50 \\
(50)\end{array}$ & $\begin{array}{c}108 \mathrm{Hg} \\
(105-110)\end{array}$ & $\frac{16}{-}$ \\
\hline 14 & 356 & $18.1 \mathrm{X}$. & $\begin{array}{l}\text { Samson } \\
(169 \mathrm{~cm})\end{array}$ & 66,550 & Hypnotischer Schlaf & $\begin{array}{c}36,0^{\circ} \\
\left(21,6^{\circ}\right)\end{array}$ & $\begin{array}{c}56 \\
(56-56)\end{array}$ & $\begin{array}{c}180 \mathrm{H}_{2} \mathrm{O} \\
-\end{array}$ & $\begin{array}{c}16 \\
(15-17)\end{array}$ \\
\hline 15 & 357 & $20 . \Gamma X$. & $"$ & 66,900 & $\begin{array}{l}\text { Hypnose mit Suggestion einer } \\
\text { schweren seelischen Depression } \\
\text { (Enucleatio bulbi und syinpathi- } \\
\text { sche Ophthalmie) }\end{array}$ & $\left(17,7^{\circ}\right)$ & $(64-78)$ & - & $\begin{array}{c}20 \\
(14-28)\end{array}$ \\
\hline$\overline{16}$ & $391 \mathrm{a}$ & 14.XII. & $"$ & $\overline{66,500}$ & Hypnotischer Schlaf & $\begin{array}{l}36,0^{\circ} \\
\left(16,7^{\circ}\right)\end{array}$ & $\begin{array}{c}63 \\
(58-70)\end{array}$ & $90 \mathrm{~mm} \mathrm{Hg}$ & $\begin{array}{c}16 \\
(15-17)\end{array}$ \\
\hline 17 & $391 b$ & 14.XII. & $"$ & 65,500 & \begin{tabular}{|ll}
$\begin{array}{l}\text { Hypnose } \\
\text { schweren }\end{array}$ & $\begin{array}{l}\text { mit Suggestion einer } \\
\text { selischen Depression } \\
\text { (Gehirntumor) }\end{array}$ \\
\end{tabular} & $\begin{array}{c}36,0^{\circ} \\
\left(16,0^{\circ}\right)\end{array}$ & $\begin{array}{c}61 \\
(58-65)\end{array}$ & $98 \mathrm{~mm} \mathrm{Hg}$ & $\begin{array}{c}16 \\
(15-17)\end{array}$ \\
\hline 18 & $393 a$ & 21.XII. & $\begin{array}{c}\text { Laufer } \\
(165 \mathrm{~cm})\end{array}$ & 67,000 & Hypnotischer Schlaf & $\begin{array}{c}36,25^{\circ} \\
\left(17,0^{\circ}\right)\end{array}$ & $\begin{array}{c}50 \\
(48-52)\end{array}$ & $90 \mathrm{~mm} \mathrm{Hg}$ & $\begin{array}{c}17 \\
(15-19)\end{array}$ \\
\hline 19 & $393 b$ & 21.XII. & $"$ & 67,000 & $\begin{array}{c}\text { Hypnose mit Suggestion einer } \\
\text { schweren seelischen Depression } \\
\text { (Gehirntumor) }\end{array}$ & $\begin{array}{c}36,25^{\circ} \\
\left(18,2^{\circ}\right)\end{array}$ & $\begin{array}{c}50 \\
(49-52)\end{array}$ & $90 \overline{\mathrm{mm} \mathrm{Hg}}$ & $\begin{array}{c}18 \\
(16-22)\end{array}$ \\
\hline
\end{tabular}

von $0,3 \%$. Die Zahlen mit einem Mittelwert von $+0,16 \%$ weichen so wenig von $0 a b$, daß wohl in allen Fällen die Fehlergrenzen der biologischen Methode nicht überschritten worden sind. Mithin läßt sich sagen, daß die Versuche bei L. das Ergebnis hatten, daß durch die Suggestion eines Depressionszustandes keinerlei sichere Veränderungen des respiratorischen Gaswechsels herbeigeführt wurden.

Merkwürdigerweise fielen die Versuche bei Sa mson ganz entgegengesetzt aus. Bei ihm liegen zwei Versuche vor, Nr. 15 mit Suggestion des Verlustes eines Auges und sympathischer Ophthalmie mit anschließender völliger Erblindung (vgl. Protokoll im Anhang), sowie Nr. 17 mit der hypnotischen Suggestion eines schweren organischen Hirnleidens (Hirntumor). Im ersten Falle kam es zu einer Steigerung von $12,3 \%$ gegenüber dem zwei Tage vorher angestellten Versuch mit hypnotischen Schlaf allein, im zweiten Falle betrug die Steigerung 6,4\% verglichen mit dem unmittelbar voraufgehenden Basisversuch. Dieser 


\begin{tabular}{|c|c|c|c|c|c|c|c|c|}
\hline 11 & 12 & 13 & 14 & 15 & 16 & 17 & 18 & 19 \\
\hline Verhalten der Motilität & $\begin{array}{c}1 \mathrm{CO}_{\mathbf{2}^{-}} \text {Bildung } \\
\text { im Versuch. } \\
{\left[120^{\prime}\right]} \\
\text { (com pro kg } \\
\text { und Minute) }\end{array}$ & $\begin{array}{l}\mathrm{IO}_{2} \text {-Ver- } \\
\text { brauch imVer- } \\
\text { such. [120'] } \\
\text { (cem pro kg } \\
\text { und Minute) }\end{array}$ & R.-Q. & $\begin{array}{l}\mathrm{g} N \text { im Ver- } \\
\text { such (cem } \\
\text { Urin) auf } 2 \\
\text { Stunden } \\
\text { umge- } \\
\text { rechnet }\end{array}$ & $\begin{array}{l}\text { Calo- } \\
\text { rienpro- } \\
\text { duktion } \\
\text { im Ver- } \\
\text { such }\end{array}$ & $\begin{array}{c}\text { Veränderung in } \\
\text { Proz.gegenuber } \\
\text { dem Vergleichs- } \\
\text { versuch }\end{array}$ & $\begin{array}{c}\text { Calo- } \\
\text { rien } \\
\text { pro kg } \\
\text { und } 2 \\
\text { Stun- } \\
\text { den }\end{array}$ & $\begin{array}{l}\text { Bemer- } \\
\text { kungen }\end{array}$ \\
\hline \multirow{2}{*}{$\begin{array}{l}\text { Nur zeitweise Bewegungen } \\
\text { mit dem rechten Uaumen. } \\
\text { Einmal auch mit den Bei- } \\
\text { nen. Öfter Gähnen }\end{array}$} & $\begin{array}{c}20,381 \\
(2,625 \mathrm{ccm})\end{array}$ & $\begin{array}{c}23,1 \mathrm{l} \\
(2,98 \mathrm{ccm})\end{array}$ & 0,882 & $\begin{array}{c}0,498 \mathrm{~g} \\
(95 \mathrm{ccm})\end{array}$ & 112,6 & $-5,3 \%$ & 1,74 & - \\
\hline & $\begin{array}{c}22,9+1 \\
(2,95 \mathrm{ccm})\end{array}$ & $\begin{array}{c}26,9 \mathrm{l} \\
(3,465 \mathrm{ccm})\end{array}$ & 0,852 & $\begin{array}{l}0,595 \mathrm{~g} \\
(93 \mathrm{~cm})\end{array}$ & 130,7 & $\begin{array}{l}+9,9 \% \text { ge- } \\
\text { genüber dem } \\
10 . \text { Versuch }\end{array}$ & 2,02 & - \\
\hline $\begin{array}{l}\text { Einmaliges spontanes Kopf- } \\
\text { nicken }\end{array}$ & $\begin{array}{c}22,871 \\
(2,86 \mathrm{ccm}) \\
\end{array}$ & $\begin{array}{c}26,7 \mathrm{l} \\
(3,34 \mathrm{ccm}) \\
\end{array}$ & 0,857 & $\begin{array}{c}0,9365 \mathrm{~g} \\
(300 \mathrm{ccm})\end{array}$ & 127,9 & - & 1,91 & - \\
\hline $\begin{array}{l}\text { Auf Grund der suggestionen } \\
\text { öfters schmerzhaftes Ver- } \\
\text { ziehen des Gesichtes }\end{array}$ & $\begin{array}{c}26,32 \mathrm{l} \\
(3,28 \mathrm{~cm})\end{array}$ & $\begin{array}{c}30,0 \mathrm{l} \\
(3,74 \mathrm{ccm})\end{array}$ & 0,877 & $\begin{array}{c}1,212 \mathrm{~g} \\
(380 \mathrm{~cm})\end{array}$ & 143,6 & $\begin{array}{c}+12,3 \% \text { ge- } \\
\text { gentiber dem } \\
14 . \text { Versuch }\end{array}$ & 2,15 & - \\
\hline $\begin{array}{c}\text { Zweimal Bewegen des } \\
\text { Kopfes }\end{array}$ & $\begin{array}{r}21,68 \mathrm{I} \\
(2,76 \mathrm{ccm}) \\
\end{array}$ & $\begin{array}{c}24,81 \\
(3,16 \mathrm{ccm}) \\
\end{array}$ & 0,874 & $\begin{array}{c}0,592 \mathrm{~g} \\
(88 \mathrm{ccm}) \\
\end{array}$ & 120,2 & - & 1,835 & - \\
\hline $\begin{array}{c}\text { Einmal Umsehen beim Auf- } \\
\text { wachen ; sonst völlige Kör- } \\
\text { perruhe }\end{array}$ & $\begin{array}{c}21,71 \mathrm{l} \\
\left(115^{\prime}\right) \\
(2,88 \mathrm{~cm}) \\
\end{array}$ & $\begin{array}{c}2.581 \\
\left(115^{\prime}\right) \\
(3,43 \mathrm{ccm}) \\
\end{array}$ & 0,842 & $\begin{array}{c}0,517 \mathrm{~g} \\
(83 \mathrm{ccm})\end{array}$ & 127,9 & $+6,4 \%$ & 1,95 & - \\
\hline $\begin{array}{l}\text { Während des ganzen Ver- } \\
\text { suches völlige Körperruhe }\end{array}$ & $\begin{array}{c}22,379 \mathrm{l} \\
(2,783 \mathrm{ccm})\end{array} \mid$ & $\begin{array}{c}27,01 \\
(3,358 \mathrm{ccm}) \\
\end{array}$ & 0,829 & $\begin{array}{c}0,7635 \mathrm{~g} \\
(135 \mathrm{ccm})\end{array}$ & 129,4 & - & 1,93 & - \\
\hline $\begin{array}{l}\text { Einzelne Bewegungen mit } \\
\text { den Lippen, als ob sie zu } \\
\text { trocken wären }\end{array}$ & $\begin{array}{c}22,2781 \\
(2,771 \mathrm{ccm})\end{array}$ & $\begin{array}{c}26,9 \mathrm{l} \\
(3,346 \mathrm{ccm})\end{array}$ & 0,828 & $\begin{array}{c}0,885 \mathrm{~g} \\
(200 \mathrm{ccm})\end{array}$ & 129,0 & $-0,3 \%$ & 1,925 & - \\
\hline
\end{tabular}

letztere Versuch wurde allerdings dadurch etwas gestört, da $\beta$ der Kranke kurz vor der Beendigung unter dem Druck einer bevorstehenden Verabredung erwachte und sich umsah. Allerdings schlief er dann unter dem Einfluß einer neuen Suggestion sofort wieder ein, so daß nicht anzunehmen ist, daß eine nennenswerte Störung des Versuches auf diese Weise eingetreten ist. Bei $\mathbf{S}$. bestand vollständige Amnesie, so daß jedenfalls subjektive Störungen nicht bewußt geworden sind. Im Gegensatz zu den Versuchen an L. ist also hier beide Male eine Stoffwechselsteigerung dagewesen. Die etwas niederen Werte im zweiten Versuch könnte man damit erklären, daß entsprechend dem Versuche bei körperlicher und geistiger Arbeit ein gewisses Training bzw. eine Anpassung eingetreten war.

Es ist schwer, sicher zu sagen, warum die beiden Versuchsreihen zu entgegengesetztem Resultat führten. In der äußeren Anlage der Versuche, insbesondere im Verhalten der Motilität, des Blutdruckes sowie 
des Pulses und der Atmung finden sich keine Anhaltspunkte, die eine eindeutige Erklärung des differenten Verhaltens gestatten. Wenn wir den Ausfall auf individuelle Momente zurückführen, so ist damit natürlich nichts gesagt. Möglicherweise liegt der Grund darin, daß unter dem Einfluß der angegebenen Störungen die Depressionen bei L. nicht so schwer und stark gewesen sind wie in den Versuchen bei Samson, der sich anscheinend besser für solche Versuche eignete. Der negative Ausfall ist natürlich immer weniger beweiskräftig wie ein positiver Befund, weil durchaus die Möglichkeit besteht, daß der Hypnotisierte in dem einen oder anderen Falle irgendwie bewußt oder unbewußt durchschaut, daß es sich nicht um volle traurige Wirklichkeit, sondern nur um eine Vortäuschung und ein Spiel handelt. Tatsächlich haben sich ja auch bei L. Gegensuggestionen eingestellt und ihm selbst bemerkbar störend geltend gemacht. Es ist sehr wahrscheinlich, daß ein schwerer hypnotisch erzeugter Depressionszustand seelisch nicht gleichwertig einem wirklichen ist. Selbstverständlich ist eine Versuchsreihe mit positiven Resultaten nicht geeignet, das in Frage stehende Problem zu lösen, und es wäre wünschenswert, diese Versuche auf einen größeren Kreis von Versuchspersonen auszudehnen. Allerdings dürften solchen Untersuchungen an einem größeren Materiale sehr erhebliche Schwierigkeiten im Wege stehen. Vor allem dürfte es nicht leicht sein, viele Versuchspersonen zu finden, die ihre Zustimmung żu derartigen Suggestionen geben. Da wir selbst leider vorläufig nicht in der Lage sind, diese Untersuchungen fortzusetzen ${ }^{1}$ ), möchten wir die bisherigen Resultate mitteilen, auch wenn sie anscheinend einander widersprechen, um so mehr, als wir überzeugt sind, daß der beschrittene Weg an einem größeren Materiale zum Ziele führen wird und schließlich eine ein deutige Antwort gestattet. Die angewandte Methodik scheint jedenfalls zur Inangriffnahme solcher Fragen fein genug. Eine Diskussion über die Ursachen der Stoffwechselsteigerung bei S. verbietet sich vorläufig noch.

Außer den geschilderten Versuchen wurde bei L. noch eine weitere Frage studiert. Es wurde geprüft, ob die Vorstellung einer sehr intensiven körperlichen Arbeit auf hypnotischem Wege zu einer Steigerung des respiratorischen Gaswechsels führt, ohne daß es zu einer Muskelbewegung selbst kommt. Der Einfluß körperlicher Vorgänge auf Puls, Blutdruck und Atmung auf das Volumen einzelner Körperglieder ist in den letzten Jahrzehnten sehr eingehend von verschiedenen Autoren untersucht worden. Erwähnt seien vor allem die umfassenden Versuche von A. Lehmann ${ }^{2}$ ) und seinen Schülern, sowie aus der allerletzten

1) Anmerkung bei der Revision: Inzwischen sind solche Versuche wieder von neuem aufgenommen worden.

2) Die körperlichen Äußerungen psychischer Zustände, 1. Teil Plethysmograph. Untersuchungen. Leipzig, Reisland 1899. 
Zeit die Untersuchungen von Weber $^{1}$ ). Gerade aus den Weberschen Versuchen geht hervor, daß bei einer auf hypnotischem Wege erzeugten Vorstellung das Volumen des betreffenden Körperteils, das bewegt werden soll, z. B. des Armes, ganz erheblich zunahm. Es wäre nun möglich, daß diese vermehrte Durchblutung der betreffenden Körperteile gleichzeitig auch mit einer Steigerung des Stoffwechsels verknüpft wäre.

Als Suggestion schwerer körperlicher Anstrengung kam vor allen Dingen Rudern, Radeln und Stemmen schwerer Gewichte usw. in Betracht.

Für die Frage, ob die Suggestion einer schweren körperlichen Anstrengung bei vollkommen ruhendem Körper den Stoffwechsel steigert, sind die Versuche Nr. 3, 7 und 11 verwertbar. Als Beispiel für die Versuchsdurchführung ist im Anhange das Protokoll von Nr. 7 mitgeteilt. Im ersteren Falle wurde eine Steigerung von etwa 12,4\% beobachtet. Dieser Versuch ist darum jedoch nicht ganz beweiskräftig, weil einmal in dem voraufgehenden Basisversuch nur die Kohlensäureausscheidung bestimmt wurde. Noch schwerer fällt ins Gewicht, daß bei diesem Versuch eine recht erhebliche Beschleunigung der Atmung bis zu 48 (Stab 10) eintrat, so daß mit großer Wahrscheinlichkeit die relativ hohe Steigerung des Stoffwechsels auf die Vermehrung der Atmung zurückzuführen ist. Eine weitere Störung wurde durch starken Harndrang und nicht vollständig körperliche Ruhe bedingt. Merkwürdig ist, daß in diesem Versuch eine ungewöhnliche große Menge Harn entleert wurde $(655 \mathrm{ccm}$ in 2 Stunden) ohne reichlichere Flüssigkeitsaufnahme vorher. Im zweiten Versuche betrug die Steigerung des Stoffwechsels nur noch 3,8\%, im dritten 1,7\%. In beiden Fällen stieg die Respiration nur bis maximal 30 an. Diese 3,8 und 1,7\% entfernen sich nur so wenig von der Nullinie, daß sie zumal im Hinblick auf die Steigerung der Atemfrequenz wohl als in die Fehlerquellen der Methoden hereinfallend betrachtet werden können. $\mathrm{Ob}$ es ein Zufall ist, daß die Werte auch hier wieder gradatim herabgehen, von 12,8 auf $1,7 \%$, oder ob auch hier wieder eine Gesetzmäßigkeit etwa im Sinne einer Gewöhnung und Anpassung vorliegt, ist außerordentlich schwer zu entscheiden. Jedenfalls haben die Versuche ergeben, daß ein sicherer Einfluß der seelischen Vorstellung einerschweren körperlichen Arbeit auf den Stoffwechsel nicht vorliegt.

Betrachten wir die Werte der respiratorischen Quotienten, so fällt auf, daß diese in den Hypnoseversuchen bis zum 8. Versuch im

1) Der Einfluß psychischer Vorgänge auf den Körper, insbesondere auf die Blutverteilung. Springer, Berlin 1910. 
Durchschnitt beträchtlich höher war als bei den folgenden Versuchen. Dies ist darauf zurückzuführen, daß am Abend vorher verhältnismäßig reichliche Kohlenhydratnahrung, wie dies bei den heutigen Ernährungsverhältnissen die Regel ist, aufgenommen wurde. Aus neueren Versuchen von Benedict ${ }^{1}$ ) ist bekannt, daß, falls am Abend reichliche Mengen von Kohlenhydraten aufgenommen werden, der respiratorische Quotient noch am folgenden Morgen stark erhöht ist.

Uber den Einfluß der verschiedenen Suggestionen auf den Blutdruck geben die Versuche kein klares Resultat. Nur bei dem 7. Versuche trat gegenüber dem Vergl ichsversuch anscheinend eine beträchtliche Steigerung zutage und im Versuch Nr. 3 stieg der Blutdruck von 160 auf 180 und von 150 auf $\mathrm{cm} 165 \mathrm{H}_{2} \mathrm{O}$. Wir möchten diesen Ergebnissen jedoch aus dem oben S. 239 angegebenen Grunde keine allzu große Beweiskraft zubilligen.

Ein gewisses Interesse hat auch das Verhalten von Puls und Atmung während der Versuche. Im allgemeinen wurde stets die Beobachtung gemacht, daß während der Hypnose der Puls sinkt, und zwar nicht nur gegenüber dem Normalzustand, sondern auch gegenüber der vorhergeh $€$ nden Hypnose, so daß die Werte am Ende des Versuches deutlich niedriger liegen als vor und nachher bei körperlicher Ruhe. Vergleicht man die Mittelwerte ${ }^{2}$ ) der Pulsfrequenz während der ersten Reihe von Untersuchungen, 3.-13. September, so gehen die Werte sukzessive von 60 auf 49 herab. Die zweite Reihe, 22.-25. September, setzt dann wieder mit 54 ein und geht langsam auf 50 zurück. Bei Samson kam diese Eigentümlichkeit weniger deutlich zum Ausdruck, jedoch liegen dafür zu wenig Versuche bei ihm vor. Ein einziges Mal gelang es bei der Vorstellung schwerer körperlicher Arbeit im Versuch Nr. 3, die Pulsfrequenz von 48 auf 68 vorübergehend zu erhöhen. In den Versuchen mit Suggestion einer starken körperlichen Anstrengung war die Atmung stets erhöht bis maximal 48. Während der Versuche mit hypnotischer Suggestion einer schweren Depression fand sich im Durchschnitt fast regelmäßig auch ein geringer aber deutlicher Anstieg der Atemfrequenz, im Durchschnitt um 2-3 Züge in der Minute.

Die Harnverhältnisse sind, da es sich nur um Zweistundenversuche handelt, natürlich außerordentlich schwer zu beurteilen. Die Werte für die $\mathrm{N}$-Ausscheidung schwanken in ziemlich weiten Grenzen, auch wenn man den ganz aus der Reihe fallenden Wert des ersten Versuches außer Betracht läßt. Im allgemeinen bewegen sich die Zahlen um 1,0 bis 0,7 herum. Sichere Unterschiede zwischen den einzelnen Ver-

1) F. G. Benedict, L. E. Emmes und J. A. Riche. Americ. journ. of physiol. 27, 383. 1910-11.

2) Gewonnen aus etwa 10-15 Einzelzählungen. Stets wurde mindestens $1 / 2$ Minute gezählt. 
suchsarten bestanden nicht. Einmal waren die Werte bei der Depression höher als im Vergleichsversuch, das andere Mal niedriger. Das gilt auch für die Versuche, bei denen sich der Depressionsversuch unmittelbar an den Grundversuch angeschlossen hat. Von größter Bedeutung für die Höhe des Eiweißumsatzes ist ja stets der unkontrollierte N-Gehalt der Nahrung am Vortage.

Da in der Literatur über Hypnose ${ }^{\mathbf{1}}$ ) die Frage nach dem schädigenden Einflusse häufiger und langer Hypnosen manchmal ${ }^{2}$ ) erörtert wird, so möchten wir am Schlusse noch erwähnen, daß die zirka 70 zum Teil stundenlangen Hypnosen bei beiden Herren ihren Gesundheitszustand sowie ihre Arbeitskraft nicht beeinflußt haben. Bei L. trat eine geringe Abnahme des Körpergewichtes auf, die aber auch andere Gründe haben kann, da L. in den Versuchswochen sich zum Staatsexamen vorbereitete, das unmittelbar nach Beendigung der Versuche begann.

Insbesondere sei darauf hingewiesen, daß der Inhalt der Depressionen beiden Herren niemals, auch nicht in Traumvorstellungen, störend zum Bewußtsein gekommen ist.

\section{Anhang: Beispiele für die Versuchsdurchführung.}

Versuch Nr. 4 (Laufer).

6. IX. 1919. Hypnose mit der Suggestion einer schweren psychischen Depression (Dr. Traumann).

Vor der Hypnose Puls 64, Atm. 15, Blutdruck 170 cem $\mathrm{H}_{2} \mathrm{O}$, Temp. 36,4 ${ }^{\circ}$.

7 Uhr 30 Min. Beginn der Hypnose. Positive Halluzination auf entsprechende Suggestion. Hören von Glockenläuten. Die Körpermuskulatur ist vollständig erschlafft.

Suggestion: Untersuchung, Herz und Lungen sind gesund, in der Magengegend ist eine Resistenz fühlbar. Es wird eine Durchleuchtung vorgenommen und dabei an der kleinen Kurvatur ein Magencarcinom festgestellt.

7 Uhr 50 Min. Einfahrt in den Kasten. Bestätigung des Röntgenbildes durch Prof. Gra fe. Erwartungsspannung, ob opera bel oder nicht. Bescheid: nicht operabel.

8 Uhr Puls 48, Atm. 20, Blutdruck 170.

8 Uhr 15 Min. Puls 52, Atm. 18, Blutdruck 170.

8 Uhr 20 Min. Puls 52, Atm. 19, Blutdruck 170.

Suggestion: große Sorgen über die bevorstehenden Qualen und über die Aufgabe des Berufes.

8 Uhr 30 Min. Suggestion: immer größerer Sorgen, L. muß als cand. med. genau wissen, was ihm bevorsteht. Trostloser Zustand. Puls 52, Atm. 24.

8 Uhr 40 Min. trostloser Zustand, das Leiden schreitet immer weiter vor.

9 Uhr Suggestion: bleiches kachektisches Aussehen. Trockene Zunge, hoff-

1) Vgl. z. B. Der Hypnotismus, seine psychologische, psycho-physiologische und therapeutische Bedeutung von A. Forel, 7. Aufl. Enke, Stuttgart 1918.

2) Vgl. darüber die Arbeiten im Journ. f. Physiol. u. Neurol. zugl. Zeitschr. f. Hypnotismus, herausgeg. von August Forel und Oscar Vogt. Ambrosius Barth, Leipzig. Eingehende Literatur bei A. Moll, Hypnotismus. Berlin 1907 u. F. Mohr im Handbuch der Neurol. I. 2. 8. 1573.1910. 
nungsloser Zustand. Metastasenbildung bereits nachweisbar. Puls 52, Atm. 19, Blutdruck 170.

9 Uhr 15 Min. Suggestion: Kummer und Sorge, auch keine Bestrahlung hilft mehr.

9 Uhr 20 Min. weitere Metastasenbildung. „Sie sind ganz schwach, bewegungslos, das Herz klopft stark." Sie haben schwere Sorgen. Auf Befragen, ob er sich elend fühle, nickt er mit dem Kopfe. Puls 52, Atm. 20, Blutdruck 170.

$9 \mathrm{Uhr} 30 \mathrm{Min}$. weitere Suggestion des hoffnungslosen Zustandes.

9 Uhr 40 Min. Puls 50, Atm. 19. Suggestionen werden weiter gegeben.

9 Uhr 50 Min. Abstellen der Gasuhr.

9 Uhr 53 Min. Ausfahrt aus dem Kasten.

$10 \mathrm{Uhr} 10 \mathrm{Min}$. Suggestion: Sie sind ganz gesund, alles war nur ein Traum. Sie fühlen sich nach der Hypnose frisch und munter. Nach dem Aufwachen Amnesie. In den Reinen etwas Müdigkeit. Puls 48, Atm. 16, Blutdruck 190.

La ufer: Ich erinnere mich der Untersuchung durch Dr. Tra umann. Ich glaubte nicht so recht an diese, da sie in zu kurzer Zeit stattfand. Weiter wurde mir suggestiert, ich hätte ein Magencarcinom und müßte als cand. med. wissen, was das bedeute. Sofort kam mir die Gegensuggestion, da $B$ ich als bis dahin so gesunder junger Mensch kaum so schnell ein derartiges Leiden bekommen könnte. Auch an die schnelle Metastasenbildung glaubte ich nicht sofort. Erst langsam glaubte ich an die Realität der Suggestionen. Kurz nach dieser Hypnose bestand Amnesie, erst später erfuhr ich, daß mir die Suggestion gegeben war, falls Prof, Grafe mich fragen sollte, alles zu erzählen.

Versuch Nr. 15 (Samson).

20. IX. 1919. Hypnose mit Suggestion einer schweren psychischen Depression (Dr. Traumann).

Vor der Hypnose Puls 75, Atm. 14, Temp. 36.

7 Uhr 45 Min. Beginn der Hypnose.

7 Uhr 50 Min. Suggestion: Splitter im rechten Auge. Der Versuch der Entfernung gelingt nicht. Sie haben Schmerzen am rechten Auge. Auf die Frage. ob er solche habe, bejaht S. Krampfhaftes Schließen der Augen (Hypnose mit geöffneten Augen).

7 Uhr 56 Min. Einfahrt in den Kasten. Suggestion: Transport in die Augenklinik zur Operation. Sorgen um das rechte Auge, das wegen einer eitrigen Entzündung entfernt werden muß.

8 Uhr 2 Min. Suggestion: Vorbereitung zur Operation.

8 Uhr 5 Min. Suggestion: Miterleben der Operation. Schilderung der Einzelheiten bei Exenteratio bulbi. Das Auge ist nicht ganz unempfindlich. Es empfindet Schmerzen dabei. Oftmals schmerzhaftes Verziehen des Gesichtes. Puls 78, Atm. 16.

8 Uhr 10 Min. Suggestion: Folgen der Operation. Aufgabe des Berufes, auch das linke Auge muB entfernt werden. Puls 74, Atm. 28. Suggestion: die Schmerzen des linken Auges nehmen jetzt zu.

8 Uhr 15 Min. Suggestion: Erwartungsspannung über die Entscheidung, ob auch das linke Auge entfernt werden soll oder nicht.

$8 \mathrm{Uhr} 10 \mathrm{Min}$. Suggestion: Entscheidung durch Geheimrat X., das Auge muß entfernt werden. Weinerlich schmerzverzogenes Gesicht. Puls 78, Atm. 30, unregelmäßig, ungleichmäßig.

8 Uhr 25 Min. Suggestion: Miterleben der zweiten Operation. Schilderung von unangenehmen Details (Schmerzen, bedrohliche, die Operation erschwerende Komplikationen, Hinzuziehen des Ohrenspezialisten, da Gehör in Gefahr). 
8 Uhr 30 Min. Atm. 14, unregelmäßig, ungleichmäßig. Gesichtsausdruck deprimiert, schmerzhaft verzogene Gesichtszüge, Puls 78.

8 Uhr 35 Min. Suggestion: hoffnungslose Zukunft. Sie sind blind, entstellt und fast taub. Puls 72, Atm. 20.

8 Uhr 45 Min. Suggestion: von Haus schlechte Nachrichten. Erwartungsspannung, Puls 72, Atm. 18.

9 Uhr Suggestion: Brief vom Vater, das Vermögen ist verloren. Der Vater ist krank, die Familie kann den Sohn nicht besuchen, obgleich er hoffnungslos in der Klinik liegt.

9 Uhr 20 Min. Puls 64, Atm. 19, Suggestion: Lebensüberdruß. Fortwährend Schilderung der fürchterlichen Lage.

9 Uhr 50 Min. Puls 72, Atm. 20, unregelmäßig, ungleichmäßig.

$9 \mathrm{Uhr} 50$ Min. Die Gasuhr wird abgestellt. Gleich danach Aufwecken, die Suggestion: „Alles war nur ein Traum, dessen Inhalt vergessen ist, nach dem Aufwachen sind Sie ganz munter und frisch." Positive Suggestion: eine Minute nach dem Aufwachen zu sagen: „Hoffentlich gibt es heute nachmittag schönes Wetter." $\mathbf{S}$. fühlt sich heute benommen, er weiß nichts mehr von den gegebenen Suggestionen. Puls 78, Atm. 16. Nachtrag zur Hypnose: Während der Suggestionen der Operationen vereinzelt Bewegungen mit den Fingern und einmal Zurückbiegen mit dem Kopf. Auch einmal leichtes Anstemmen der Füße gegen das Fußbrett, alles offenbar Schmerzreaktionen.

Samson: Von dieser Hypnose ist mir gar nichts bekannt.

Versuch Nr. 7 (L.).

11. IX. 1919. Hypnose mit der Suggestion einer schweren körperlichen Anstrengung (Dr. Traumann).

Vor der Hypnose Puls 52, Atm. 15, Blutdruck $168 \mathrm{H}_{2} \mathrm{O}$.

Temp. 36,3. Beginn der Hypnose 7 Uhr 25 Min. Puls 50, Atm. 15, Blutdruck $168 \mathrm{H}_{2} \mathrm{O}$.

7 Uhr 35 Min. Einfahrt in den Kasten. Die Körpermuskulatur ist ganz erschlafft.

7 Uhr 43 Min. Suggestion: Gewichtheben in der Vorstellung. Angestrengter Gesichtsausdruck. Ab und zu Hochziehen der Augenbrauen, keine Bewegungen der Körpermuskulatur. Suggestion: das Gewicht beträgt 100 Pfund. Es wird mit Anstrengung in die Höhe gehoben. Wenn es oben ist, dann nicken Sie mit dem Kopfe. Dies tut L. nach 2 Minuten. Er soll das Gewicht oben halten. Nach einiger Zeit, wenn ,jetzt" gesagt wird, das Gewicht mit aller Kraftanstrengung herunterlassen mit gestrecktem Arm. Wenn das Gewicht unten ist, dann soll er mit dem Kopfe nicken. Dies geschieht nach einigen Minuten.

7 Uhr 50 Min. Puls 56, Atm. 17, Blutdruck $178\left(\mathrm{ccm} \mathrm{H}_{2} \mathrm{O}\right)$.

$7 \mathrm{Uhr} 55$ Min. Puls 52, Atm. 16, Blutdruck $180\left(\mathrm{com} \mathrm{H}_{2} \mathrm{O}\right)$.

8 Uhr 8 Min. Puls 50, Atm. 17, Blutdruck $180\left(\mathrm{ccm}_{2} \mathrm{O}\right)$.

(Messung während des Gewichthebens: das Gewicht wird dreimal gehoben und entsprechend abgesetzt. Niemals Körperbewegungen).

$8 \mathrm{Uhr} 10 \mathrm{Min}$. Nach 3 Min. Ruhepause. Suggestion: Rudern auf dem Neckar.

$8 \mathrm{Uhr} 10 \mathrm{Min}$. Puls 50, Atm. 30, Blutdruck $180\left(\mathrm{ccm} \mathrm{H}_{2} \mathrm{O}\right.$ ).

$8 \mathrm{Uhr} 15 \mathrm{Min}$. Puls 50, Atm. 24, Blutdruck $185\left(\mathrm{com} \mathrm{H}_{2} \mathrm{O}\right.$ ).

8 Uhr 15 Min. Der Daumen der rechten Hand wird bewegt.

8 Uhr 30 Min. Puls 46, Atm. 24, Blutdruck 178.

8 Uhr 47 Min. Puls 48, Atm. 23, Blutdruck 170.

9 Uhr 5 Min. Puls 49, Atm. 24, Blutdruck 170.

9 Uhr 20 Min. Puls 52, Atm. 26, Blutdruck 170.

9 Uhr 30 Min. Puls 50, Atm. 30, Blutdruck 170. 
252 E. Grafe u. E. Traunamn: Zur Frage des Einflusses psych. Depressionen usw.

Bis 9 Uhr 35 Min. Rudern. Mit Ausnahme der Daumenbewegung und verschiedentlich leichter Kopfbewegungen nach rückwärts ganz ruhig, hin und wieder Lecken der Lippen, als ob die Zunge zu trocken wäre. Während des Ruderns wurde dauernd die Aufmunterung gegeben, kräftig zu rudern. Suggestion: Sie strengen sich sehr an, Sie schwitzen, Sie rudern gegen den Strom usw."

(L. hat nicht geschwitzt).

9 Uhr 35 Min. Abstellen der Gasuhr.

9 Uhr 43 Min. Noch in Hypnose. Suggestion: tiefer Schlaf zum Ausruhen. Puls 47, Atm. 10, Blutdruck 180.

9 Uhr 50 Min. Aufwachen. Nach der Hypnose fühlt sich L. frisch und munter. Der Versuch ist seiner Meinung nach ,geglückt", was er gemacht, weiß er nicht mehr.

Laufer: Es besteht Amnesie. Mir sind keine Einzelheiten in Erinnerung. 